\title{
The Finite, Discontinuous Primitive Groups of Collineations in Four Variables.
}

\author{
By \\ H. F. Buichfendt of Stanford University, California.
}

A complete enumeration of the collineation-groups in four variables has not yet been published, although a number of important groups have been discovered and constructed by Jordan, Klein, Maschke and others*), and a general theorem concerning such groups has been given by Jordan**).

In two papers published in the "Transactions of the American Mathematical Society"***), the writer has, after Maschke, divided these groups into two categories, transitive and intransitive, and the former again into primitive and imprimitive groups (definitions follow below). These papers contain some theorems concerning the primitive groups in $n$ variables, by means of which a number may always be determined which is divisible by the orders of the different possible primitive groups of linear homogeneous substitutions of determirant 1 in $n$ variables. The complete enumeration of the primitive groups of collineations in four variables may be based on these theorems and ordinary group-theory. This has been done by the author, who submits his results in the following paper.

* For a bibliography and brief résumé of the work done in this field consult Wiman: "Endliche Grappen linearer Substitutionen", Encyklopädie der Mathematischen Wissenschaften, Bd. I, pp. 522-554.

*) Journal für Mathematik, 84 (1878), p. 89. The Theorem is as follows: every linear homogeneous group $(G)$ in $n$ variables has an abelian self-conjugate subgroup $(F)$ of order $f$, and the order of $G$ is $\lambda f$, where $\lambda$ is inferior to a fixed number which depends only apon $n$.

*) "On the Order of Linear Homogeneous Groups", First and Second Paper, Transactions of the American Math. Society, 4 (1903), pp. 387-397, and 5 (1904), pp. 310-325. These papers shall be referred to hereafter by "L-GI" and "L-GII", respectively. 
Considering the four variables $x, y, z, u$ as homogeneous coordinates of space of three dimensions, the nature of the classification referred to may be seen as follows:*)

An intransitive group leaves invariant, either each of two axial pencils of planes (as $x+\lambda y=0, z+\mu u=0$ ), or one plane $(x=0)$ and a pencil of planes not including this $(y+\lambda z+\mu u=0)$.

A transitive group has no such invariant configuration.

The transitive groups are separated into

imprimitive groups, the substitutions of which leave invariant a system of two axial pencils of planes $(x+\lambda y=0$, $z+\mu u=0)$, i. e. leave invariant each pencil or interchange them; - or leave invariant a system of four planes $(x=0$, $y=0, z=0, u=0$ );

primitive groups, having no such invariant configuration.

We shall, furthermore, distinguish between four classes of primitive groups in four variables: those which have invariant intransitive sub-groups; those which have invariant imprimitive sub-groups; primitive groups which are simple; and, finally, primitive groups having invariant primitive sub-groups.

It is well known that a collineation-group $G$ in $n$ variables of order. $g$ may or may not be represented as a linear homogeneous group in $n$ variables of order $g$. There exists, however, in all cases, a linear homogeneous group $G^{\prime}$ of order $n g$, isomorphic with $G$, containing the group $F$ of similarity-substitutions

$$
x_{i}^{\prime}=\varphi x_{i} ; \quad \varphi^{n}=1 ; \quad i=1,2, \cdots, n .
$$

The quotient-group $G / F$, of order $g$, is then simply isomorphic with the collineation-group considered. In the following analysis, the groups dealt with are always taken as linear homogeneous groups of subsitutions of determinant 1 , unless a factor of proportionality $(\rho)$ is used. The orders given are, however, the orders of collineation-groups, unless otherwise stated.

In all questions dealing with substitution-groups, the writer has received valuable assistance from Dr. W. A. Manning of Stanford University.

*) For the definitions of the remaining terms and phrases used the reader is referred to "L-GI" and "L-GI".

*) Maschke, Math. Ann. 52 (1899), p. 363, and Loewy, Transactions of the American Math. Soc., 4 (1903), p. 44, have proved that if a group of finite order leaves invariant a plane $(x=0)$, then it mast also leave invariant the pencil

$$
y+2 z+u u=0 \text {. }
$$




\section{The Primitive Groups having Invariant Intransitive Sub-groups.}

1. In "L-GII", \$§ 2-4, a general theory is developed for such groups. An isomorphism is established between two primitive groups, $G^{\prime}$ and $G^{\prime \prime}$, of two variables each. The matrices of the substitutions of the required group $G$ are then constructed ("produced") in a certain manner from the matrices of the corresponding substitutions of $G^{\prime}$ and $G^{\prime \prime}$. Let $S^{\prime}$ and $S^{\prime \prime}$ be two such corresponding substitutions, whose matrices are respectively

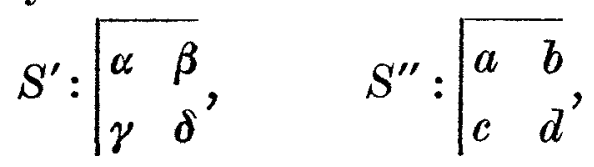

then is the matrix of the produced substitution $S$ of $G$ of the form

$$
S: \mid \begin{array}{llll}
a \alpha & a \beta & b \alpha & b \beta \\
a \gamma & a \delta & b \gamma & b \delta \\
c \alpha & c \beta & d \alpha & d \beta \\
c \gamma & c \delta & d \gamma & d \delta .
\end{array}
$$

Assuming the variables to be $x, y, z, u$, the group $G$ will, plainly, have the invariant $x u-y z=0$.

2. Now, Goursat has enumerated all the groups in 4 variables which leave this surface invariant*). In his notation, a substitution of $G$ is indicated by means of two variables, $\xi$ and $\eta$, viz:

meaning

$$
[\eta, \xi ; f(\eta), \varphi(\xi)]
$$

$$
\eta^{\prime}=f(\eta)=\frac{\alpha r+\beta}{\gamma \eta+\delta}, \quad \xi^{\prime}=q(\xi)=\frac{a \xi+b}{c \xi+d},
$$

which represent substitutions of linear fractional groups in one variable. When written in linear homogeneous form in two variables, these become our groups $G^{\prime}$ and $G^{\prime \prime}$, and the coefficients $\alpha, \beta, \gamma, \delta, a, b, c, d$ are precisely the elements $\alpha, \beta, \cdots$ in our matrix (1).

The orders of $G^{\prime}$ and $G^{\prime \prime}$, as is well known, are limited to the numbers 12, 24 and 60 . The invariant intransitive sub-group $(H)$ of $G$ can be assumed to be formed of the substitutions produced from the identical substitution of $G^{\prime \prime}$ and the substitutions of $G^{\prime}$ corresponding to this. The order of $H$ is therefore one of the numbers $4,12,24$ or 60 . It follows that the order of $G$ is one of the numbers

$$
4 \cdot 12,12 \cdot 12,4 \cdot 24,12 \cdot 24,24 \cdot 24,12 \cdot 60,24 \cdot 60,60 \cdot 60 \text {. }
$$

*) «Sur les substitutions orthogonales etc.» Annales scientifiques de l'École Normale Supérieure, (3) T. 6 (1889), pp. 9-102. 


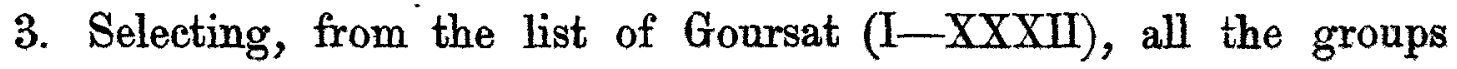
satisfying the conditions given, we have nine types, namely $X X, X X I I-X X V$ inc., XXVII-XXX inc. Of these, the groups XXII and XXVII are imprimitive, leaving invariant the function $\left(x^{2}-u^{2}\right)\left(y^{2}-z^{2}\right)$, which, by a change of variables, takes the form $x y z u$. The remaining seven types are primitive. Using his notation, these groups are as follows $\left(1^{0}-7^{\circ}\right)$ :

$1^{0}, 2^{0}, 3^{0}, 4^{0}, 5^{0}$ and $6^{0}$. Groups of orders $144,288,720,1440,576$ and 3600 respectively, represented by the symbol (2), when $[\eta, f(\eta)]$ designates any substitution of the group $A,[\xi, \varphi(\xi)]$ any one of the group $B ; A$ and $B$ being any two (alike or different) of the tetrahetral, octahedral and icosahedral groups.

$7^{0}$. Group of order 288:

$$
[\eta, \xi ; f(\eta), \varphi(\xi)][\eta, \xi ; i f(\eta), i \varphi(\xi)], \quad i^{2}=-1
$$

$[z, f(z)]$ and $[z, \varphi(z)]$ designating any two substitutions of the tetrahedral group.

4. It can readily be verified that these groups have only one invariant surface of the second degree, $x u-y z=0$. The groups containing any of the given types self-conjugately must therefore also possess this invariant surface, and are, accordingly, given by Goursat in the work referred to. Five new types must be added to our list, namely those numbered XLIII, XLV, XLVI, XLVIII and L by Goursat. They are as follows:

$8^{0}, 9^{\circ}, 10^{\circ}$ and $11^{\circ}$. Groups of orders 288, 576, 1152 and 7200 respectively, generated by the substitution

$$
[\eta, \xi ; \xi, \eta]
$$

and each of the groups $1^{0}, 7^{0}, 5^{\circ}$ and $6^{\circ}$ in turn.

$12^{\circ}$. Group of order 576 , generated by the subtitution

and the group $7^{\circ}$.

$$
[\eta, \xi ; \xi, i \eta], \quad i^{2}=-1 \text {, }
$$

On account of the single invariant surface of the second degree, $x u-y z=0$, no groups, not included in this list, can contain any of the types $1^{0}-12^{0}$ self-conjugately. In our notation, the substitution $[\eta, \xi ; \xi, \eta]$ is

$$
\varrho x^{\prime}=x, \quad \varrho y^{\prime}=z, \quad \varrho z^{\prime}=y, \quad \varrho u^{\prime}=u,
$$

$\rho$ being a factor of proportionality. The substitution $[\eta, \xi ; \xi, i \eta]$ is

$$
\varrho x^{\prime}=x, \quad \varrho y^{\prime}=z, \quad \varrho z^{\prime}=-i y, \quad \varrho u^{\prime}=-i u ; \quad i^{2}=-1 .
$$

\section{The Primitive Groups having Invariant Imprimitive Sub-groups.}

5. Such a sub-group $(H)$ has either two systems of imprimitivity, say $(x, y)$ and $(z, u)$, or it leaves invariant an equation of the form 
$x y z u=0$. In the first case, the group $H^{\prime}$ generated by the second powers of the substitutions of $H$ will be intransitive, and is obviously invariant within the required primitive group $(G)$. Consequently, $H^{\prime}$ must consist of the identical substitution alone, unless $G$ is one of the groups $1^{0}-12^{0}$.

In the second case, the letters $x, y, z, u$ are permuted according to a permutation-group $K$, which must be transitive (in the sense of transitivity of permutation-groups) when $H$ is transitive. Its order is therefore $4,8,12$ or 24 . The last two cases may be replaced by the first if for $H$ we take the group generated by the third powers of the substitutions of $H^{\prime}$, a group generated by the second powers of the substitutions of the original group $H$. If $K$ is of order 8 , then it contains the cycle $(x y z u)$, and the group $H^{\prime}$ generated by the second powers of the substitutions of $H$ is intransitive and does not reduce to the identical substitution alone. This is also true if $K$, of order 4 , contains the same cycle. Hence, we may assume that $K$ is the group $1,(x y)(z u)$, $(x z)(y u),(x u)(y z)$. But then $H$ will have two systems of imprimitivity, $(x, y)$ and $(z, u)$.

From the remark made above concerning such a group, its order must be a power of 2 . Such a group can be written in monomial*) form (Theorem 9, "L-GII"), and may readily be constructed. It is found that $H$ is of order 16 and is generated by $A, B, C$ and $D$ :

\begin{tabular}{l|c|c|c|c|} 
& $A$ & $B$ & $C$ & $D$ \\
\hline$\varrho x^{\prime}=$ & $x$ & $x$ & $y$ & $z$ \\
$\varrho y^{\prime}=$ & $y$ & $-y$ & $x$ & $u$ \\
$\varrho z^{\prime}=$ & $-z$ & $-z$ & $u$ & $x$ \\
$\varrho u^{\prime}=$ & $-u$ & $u$ & $z$ & $y$
\end{tabular}

6. This is the group given by Maschke in Mathematische Annalen 30 (1887), p. 498. To find the groups leaving $H$ invariant we may proceed according to the method of Maschke.

Let $x, y, z, u$ be homogeneous coordinates of space of three dimensions. With any two points, $\left(x_{1}, y_{1}, z_{1}, u_{1}\right)$ and $\left(x_{2}, y_{2}, z_{2}, u_{2}\right)$, will be associated a set of 6 expressions of the form (line-coordinates) called

$$
x_{1} y_{2}-x_{2} y_{1}, x_{1} z_{2}-x_{2} z_{1}, x_{1} u_{2}-x_{2} u_{1}, y_{1} z_{2}-y_{2} z_{1}, \cdots
$$

$$
p_{12}, p_{13}, p_{14}, p_{23}, \cdots
$$

*) See Maschke, American Journal of Mathematics XVII (1895), p. 168. The author has, in "L-GII", \$5, called such groups semi.canonical. 
respectively, in Maschke's paper. These expressions combine into 6 linear functions of themselves, which are relative invariants of $H$, namely

$$
\left\{\begin{array}{lll}
p_{12}+p_{34}=w_{1}, & p_{13}+p_{42}=w_{3}, & p_{14}+p_{23}=w_{5} \\
p_{12}-p_{34}=w_{2}, & p_{13}-p_{42}=w_{4}, & p_{14}-p_{23}=w_{6}
\end{array}\right.
$$

there being no other invariants of $H$, linear in $p_{12}, p_{13}, \cdots$. Moreover, $H$ contains all the substitutions which leave invariant, to constant multipliers, the functions (4), as may be proved readily. It follows that the group of order $16 \cdot 720$, (see Maschke's memoir, 1. c.) generated by $H$ and the 6 ! permutations of the expressions (4) must be itself one and contain as sub-groups all the primitive groups, if any, containing $H$ self-conjugately. The order of such a sub-group $(G)$ will be $g=16 k$, where $k$ is the order of a group $K$ of permutations of 6 letters or fewer. Moreover, any two such sub-groups whose corresponding groups $K_{1}$ und $K_{2}$ are conjugate under the symmetric group in 6 letters, can be transformed one into the other. If $K$ contains an invariant sub-group $K^{\prime}$, then will $G$ contain an invariant sub-group $G^{\prime}$, corresponding to $K^{\prime}$.

7. Now, any group $G$ of order $5 n$ will be primitive. For, it must contain a substitution of order 5 , which substitution may be transformed into the type $T$ given below (5), and we may readily prove that a group generated by $H$ and $T$ is primitive. There are in all nine substitutiongroups in not more than 6 letters $\left(w_{1}, w_{2}, w_{3}, w_{4}, w_{5}, w_{6}\right)$ of orders $5 n$, namely groups of orders $5,10,20,60,60 *), 120,120,360$ and 720 respectively, generated by $T^{\prime}=\left(w_{2} w_{3} w_{4} w_{5} w_{6}\right), T^{\prime}$ and $R_{1}^{\prime}=\left(w_{3} w_{6}\right)\left(w_{4} w_{5}\right) ; T^{\prime \prime}$ and $R_{2}^{\prime}=\left(w_{3} w_{4} w_{6} w_{5}\right) ; T^{\prime}$ and $U^{\prime}=\left(w_{3} w_{4} w_{5}\right) ; T^{\prime}$ and $W^{\prime}=\left(w_{1} w_{2} w_{3}\right)\left(w_{4} w_{5} w_{6}\right)$; $T^{\prime}$ and $M^{\prime}=\left(w_{5} w_{6}\right) ; T^{\prime}$ and $N^{\prime}=\left(w_{1} w_{2}\right)\left(w_{3} w_{4}\right)\left(w_{5} w_{6}\right) ; T^{\prime}$ and $S^{\prime}=\left(w_{1} w_{2} w_{3}\right)$; $T^{\prime}, S^{\prime}$ and $N^{\prime}$, respectively. The corresponding linear substitutions are as follows:

$R_{1}: \varrho x^{\prime}=-i y, \varrho y^{\prime}=-i x, \varrho z^{\prime}=z, \varrho u^{\prime}=u, \quad i^{2}=-1 ;$

$R_{2}: \varrho x^{\prime}=x+i y, \rho y^{\prime}=i x+y, \varrho z^{\prime}=i z+u, \varrho u^{\prime}=-z-i u$;

$S: \varrho x^{\prime}=x-u, \varrho y^{\prime}=y-z, \varrho z^{\prime}=i(y+z), \varrho u^{\prime}=i(x+u), i^{2}=-1$;

$T: \begin{cases}\varrho x^{\prime}=-x+y+i z-i u, & \varrho y^{\prime}=x+y+i z+i u, \\ \varrho z^{\prime}=-x+y-i z+i u, & \varrho u^{\prime}=x+y-i z-i u ;\end{cases}$

(5)

$V: \varrho x^{\prime}=x-y, \varrho y^{\prime}=i(x+y), \varrho z^{\prime}=z-u_{2} \varrho u^{\prime}=i(z+u)$;

$W: \begin{cases}\varrho x^{\prime}=i(x+y-z-u), & \varrho y^{\prime}=x-y+z-u, \\ \varrho z^{\prime}=i(x-y-z+u), & \varrho u^{\prime}=-x-y-z-u ;\end{cases}$

$M: \rho x^{\prime}=i z, \varrho y^{\prime}=u, \rho z^{\prime}=-x, \rho u^{\prime}=-i y$;

$N: \rho x^{\prime}=x, \rho y^{\prime}=y, \rho z^{\prime}=z, \quad \rho u^{\prime}=-u$.

*) Burnside, Theory of Groups, p. 206. 
Considering a group $G$ to which belongs a substitation-group $K$ of order $2^{a} 3^{b}$, we may first suppose, either that $K$ is simple, or that it contains a self-conjugate subgroup $K^{\prime}$ to which corresponds a non-primitive self-conjugate subgroup $G^{\prime}$ of $G$. If $K$ is simple, its order is 2 or 3 . In the first case $G$ must be of order $2^{5}$ and is then imprimitive. In the second case, $G$ must be generated by $H$ and $U$ or by $H$ and $W$. In neither case can it be primitive.

Let us next assume that $G$ has an invariant subgroup $G^{\prime}$ of order $16 \cdot 2^{a}, a \geqq 1$. Such a group can be written in monomial form, and it is found that the group $G^{\prime \prime}$, generated by the second powers of its substitutions, is intransitive and does not reduce to the identical substitution alone. Since this group $G^{\prime \prime}$ is contained self-conjugately in $G$, the latter must be found among the groups $1^{0}-7^{0}$.

Finally, let $G$ have an imprimitive invariant subgroup $G^{\prime}$ of order $16 \cdot 2^{\alpha} \cdot 3^{b}$. If this group $G^{\prime}$ contains the substitution $W$, then it will leave invariant just one function of the form $x_{1} y_{1} z_{1} u_{1}$ (namely $\left(x^{2}-z^{2}\right)\left(y^{2}-u^{2}\right)$ ) and $G$ could not be primitive, as it would leave invariant the same function. If $G^{\prime}$ contained $U$, it could not leave invariant a function of the form $x_{1} y_{1} z_{1} u_{1}$, but wonld possess two systems of imprimitivity. The group $G^{\prime \prime}$, generated by the second powers of the substitutions of $G^{\prime}$, would be intransitive, and $G$ would be found among the groups $1^{\circ}-7^{\circ}$.

8. The primitive groups other than the groups $1^{0}-12^{\circ}$ containing imprimitive self-conjugate sub-groups have now been determined and are as follows:

$13^{\circ}$. Group of order 16.5 , generated by $H$ of (3)

and $T$ of $(5)$,

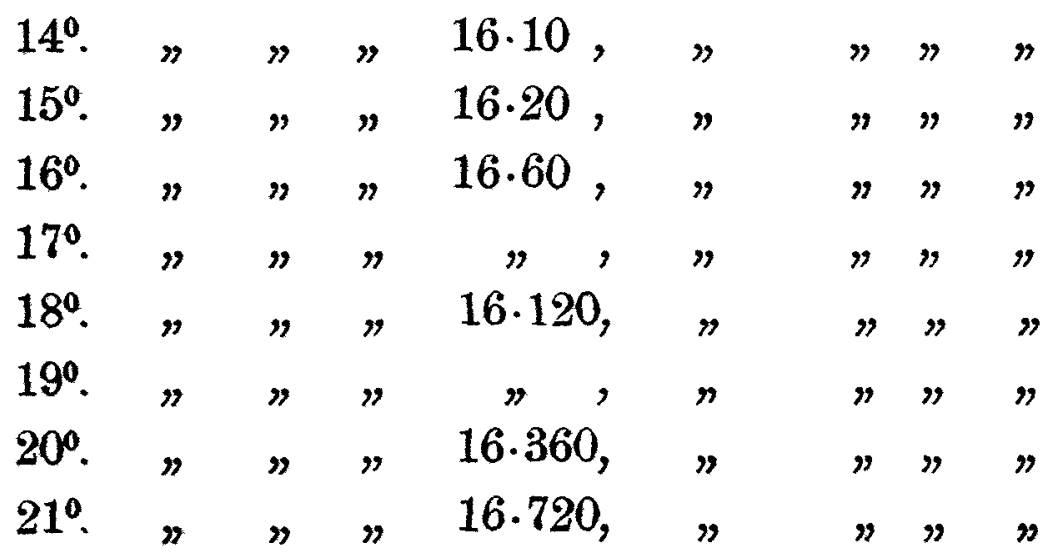

\begin{tabular}{|c|c|c|c|}
\hline$T$ & 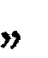 & $R_{1}$ & $"$ \\
\hline$"$ & $\eta$ & $R_{2}$ & $"$ \\
\hline$"$ & $"$ & $U$ & $"$ \\
\hline$"$ & $"$ & $W$ & $"$ \\
\hline " & $\eta$ & $M$ & $"$ \\
\hline$\eta$ & $"$ & $N$ & $"$ \\
\hline$"$ & $"$ & $S$ & " \\
\hline & $\eta$ & $N$ & $\eta$ \\
\hline
\end{tabular}

The groups $16^{\circ}$ and $17^{\circ}$ are not transformable one into the other, as $16^{\circ}$ leaves invariant one of the functions $w$ (i. e. $w_{1}$ ), and $17^{\circ}$ does not. For the same reason $18^{\circ}$ and $19^{\circ}$ are distinct types.

9. We may briefly settle the question concerning the determination of the groups containing any of these self-conjugately. The functions $w_{1}, w_{2}, \cdots, w_{6}$ satisfy the definition of being a set of six functions of the 
coordinates of two points, $x_{1}, y_{1}, z_{1}, u_{1} ; x_{2}, y_{2}, z_{2}, u_{2}$, linear in both, vanishing when the points coincide, and being left invariant, or mutually interchanged (to some constant factors), by the substitutions of the group $G$, this being any one of the groups $13^{\circ}-21^{\circ}$. Now, we may ask if there are other sets of six functions satisfying these conditions? The answer is no, as the reader may verify. It follows that if a group $G$ contains self-conjugately any of the groups $13^{\circ}-21^{\circ}$, then $G$ will permute among themselves the functions $w_{1}, w_{2}, \cdots, w_{6}$. Accordingly, as it contains $H$, it must be one of the groups $13^{0}-21^{\circ}$ already determined.

\section{The Primitive Groups which are Simple.}

10. Notation and Theorems. The notation and theorems given in "L-GI" and "L-GII" will generally be used here without further comment. For the sake of brevity and clearness, a symbolic notation will be employed, and two of the theorems referred to will be restated. We shall assume that the substitutions considered are of determinant 1 .

If the multipliers of a substitution $S$ are $\alpha, \beta, \gamma, \delta$, we shall say that $S$ is of type $(\alpha, \beta, \gamma, \delta)$. The group $K$ generated by $S$ is said to be of type $(\alpha, \beta, \gamma, \delta)$.

The scheme

$$
G=\begin{array}{c|c|c|c}
x & y & z & u \\
\hline S: \alpha_{1} & \beta_{1} & \gamma_{1} & \delta_{1} \\
T: \alpha_{2} & \beta_{2} & \gamma_{2} & \delta_{2} \\
\cdot & \cdot & \cdot & \cdot \\
\hline
\end{array}
$$

implies that $S, T, \cdots$ are substitutions of, or generate, an abelian group $G$, written in canonical form. More particularly, we have

$$
S: x^{\prime}=\alpha_{1} x, y^{\prime}=\beta_{1} y, z^{\prime}=\gamma_{1} z, u^{\prime}=\delta_{1} u ; \text { etc. }
$$

The scheme

\begin{tabular}{cc|c|c}
$x$ & $y$ & $z$ & $u$ \\
\hline$S: \alpha_{1}$ & $\beta_{1}$ & $\gamma_{1}$ & $\delta_{1}$ \\
$T: \alpha_{2}$ & $\beta_{2}$ & $\gamma_{2}$ & $\delta_{2}$ \\
$\cdot$ & $\cdot$ & $\cdot$ & $\cdot$ \\
\hline
\end{tabular}

implies that $S, T, \cdots$ are substitutions of an intransitive group, the systems of intransitivity being $(x, y), z$ and $u$. In general, the vertical bars will separate the systems of intransitivity.

The symbol $G_{n}$ means "group(s) of order $n$ ", and $G_{n}^{\prime}$ "the number 
of sub-groups of order $n$ ". Similarly, $S_{n}$ means "substitution(s) of order $n$ ", and $S_{n}^{\prime}$ "the number of substitutions of order $n$ ".

11. Theorem $10^{*}$ ). If a group $G$ has a substitution $S$ of variety $m$ and of order $p^{a+c} \geqq m p^{c}$, then will $G$ contain a self-conjugate subgroup $H$ containing $S^{p^{a}}$. Any substitution $T$ of $H$ will possess the property $(V)_{p} \equiv(V T)_{p}(\bmod . p), V$ being any substitution of $G$. In particular, $4 \equiv(T)_{p}$ (mod. $\left.p\right)$.

The letter $H$ will throughout be reserved for a sub-group of this character. As we are now considering simple groups only, it is clear that if we have a substitution of the kind stated, then must $G=H$.

According to this theorem we find that we can have no linear substitutions of orders 32,27 or $p^{2}, p \geqq 5$, unless we get $H$. Excluding the group $H$, the only types of $S_{8}, S_{16}$ and $S_{9}$ (linear substitutions) are found to be

$$
\begin{array}{ll}
S_{8}:(\alpha, \alpha, \alpha,-\alpha),(\alpha,-\alpha, i \alpha,-i \alpha) ; & \alpha^{2}=i, i^{2}=-1 . \\
S_{16}:(\beta, \beta,-\beta, i \beta) ; & \beta^{2}=\alpha . \\
S_{9}: \begin{cases}\left(\omega^{2}, \varphi, \varphi \omega, \varphi \omega^{2}\right),\left(\varphi, \varphi^{2}, 1, \omega^{2}\right), & \\
\left(\varphi, \varphi^{2} \omega^{2}, \omega, \omega^{2}\right),\left(\varphi, \varphi \omega, \varphi^{2} \omega, \varphi^{2} \omega^{2}\right), & \varphi^{3}=\omega, \omega^{3}=1 ; \omega \neq 1 .\end{cases}
\end{array}
$$

12. The types of abelian groups of orders $2^{a}$ and $3^{a}$ are limited by Theorem 10 and an obvious extension to this theorem. Thus, if $G$ contains the sub-group generated by

\begin{tabular}{cc|c|c|c} 
& \multicolumn{1}{c}{$x$} & $y$ & $z$ & $u$ \\
\cline { 2 - 3 }$T:$ & 1 & 1 & -1 & -1 \\
$S:$ & $i$ & $-i$ & 1 & 1 \\
$R:$ & $i$ & 1 & $-i$ & 1 \\
$U:$ & $i$ & 1 & 1 & $-i$, \\
\hline
\end{tabular}

then $G$ will contain a group $H^{*}$. To show this, form the determinant corresponding to the determinant (6) of "L-GI", with $(V),(V T),(V S)$, $(V R)$ and $(V U)$ for the elements of the first column, $\nabla$ being any substitution of $G$. After reduction we find the equation

$$
(V)-(\nabla T)+2 i[(V T)+(\nabla S)]-(1+i)[(V R)+(V U)]=0
$$

from which follows

$$
(V)_{2}-(V T)_{2} \equiv 0(\bmod .2),
$$

the equation eharacteristic for the substitutions $(T)$ of the group $H$.

*) "L-G II", § 6. 
13. Assuming that $G$ does not contain $H$, the abelian sub-groups of $G$ (considered as a limear homogeneons group) of orders $2^{a}$ may now be constructed without much difficulty. We may suppose that such a group $G^{\prime}$, when written in linear homogeneous form, will always contain the group $G_{4}$ of similarity-substitutions. Then we find that the order of the quotient group $\frac{G}{G_{1}}$ is not greater than 8 . Referring now to Theorem 10 we see that the highest power of 2 which divides the order of a collineationgroup is $2^{6}$, unless the group contains $H$.

Under the same restriction, we may construct all types of sub-groups of order $3^{a}$. We shall give them here for future reference.

(a) Groups of order 3:

$$
(1, \omega, \omega, \omega),\left(1,1, \omega, \omega^{2}\right),\left(\omega, \omega, \omega^{2}, \omega^{2}\right) ; \quad \omega^{3}=1 .
$$

( $\beta$ ) Groups of order 9:

\begin{tabular}{|c|c|c|c|c|c|c|c|c|c|c|c|}
\hline 1 & $\boldsymbol{\omega}$ & $\omega$ & $\omega$ & 1 & $\boldsymbol{\omega}$ & $\omega$ & $\omega$ & 1 & 1 & $\boldsymbol{\omega}$ & $\omega^{2}$ \\
\hline 1 & 1 & $\omega$ & $\omega^{2}$ & 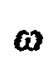 & 1 & $\omega$ & $\omega$ & $\boldsymbol{\omega}$ & $\omega^{2}$ & 1 & 1 \\
\hline
\end{tabular}

and the four types given under $S_{9}$ in (6).

(y) Groups of order 27:

\begin{tabular}{|c|c|c|c|c|c|c|c|c|}
\hline 1 & $\omega$ & $\omega$ & $\omega$ & & 1 & $\omega$ & $\omega$ & \\
\hline$\varphi$ & $\varphi^{2}$ & 1 & $\omega^{2}$, & $\varphi^{3}=\omega$ & $\omega$ & 1 & $\omega$ & \\
\hline
\end{tabular}

and two groups generated by

$$
U: x^{\prime}=x, y^{\prime}=z, z^{\prime}=u, u^{\prime}=y
$$

and $A$ and $B$ respectively, where

$$
A=\begin{array}{l|l|l|l|l}
x & y & z & u \\
\hline 1 & \omega & \omega & \omega \\
1 & 1 & \omega & \omega^{2}
\end{array} \quad \quad B=\begin{array}{ll|l|l|l|l}
x & y & z & u \\
\hline \omega^{2} & \varphi & \varphi \omega & \varphi \omega^{2}
\end{array}
$$

(d) Group of order 81 generated by $U$ and

\begin{tabular}{l|l|l|l}
$x$ & $y$ & $z$ & $u$ \\
\hline 1 & 0 & $\omega$ & $\omega$ \\
$\omega$ & 1 & $\omega$ & $\omega$ \\
$\omega$ & 0 & 1 & $\omega$ \\
\hline
\end{tabular}


The following statement may now be made: If $G$ does not contain $H$, the highest power of 3 that may divide the order of $G$ is $3^{4}$.

14. Theorem 11.*) Let $G$ contain two permutable substitutions, $S$ and $T$, of different prime orders $p \neq 2$ and $q$, generating the group

$$
\begin{array}{ll|l|l|l}
S: & \alpha_{1} & \alpha_{2} & \alpha_{3} & \alpha_{4} \\
T: & \beta_{1} & \beta_{2} & \beta_{3} & \beta_{4}
\end{array} \quad \quad \quad \alpha_{i}^{p}=1, \beta_{i}^{q}=1 .
$$

Now, if $\beta_{1} \neq \beta_{2} \neq \beta_{3} \neq \beta_{4}$ and $q>4$, then will $G$ contain the group $H$. This would also be the case if $\beta_{1}=\beta_{2} \neq \beta_{3} \neq \beta_{4}, \alpha_{1}=\alpha_{2}, q \geqq 3$; or if $\beta_{1}=\beta_{2}, \beta_{3}=\beta_{4}, \beta_{1} \neq \beta_{3}, \alpha_{1}=\alpha_{2}, \alpha_{3}=\alpha_{4}, q \geqq 2$; etc. - An evident extension to this theorem is obtained by considering a possible abelian group of order $p q^{a}$, as in the case

\begin{tabular}{c|c|c|c}
\hline$\alpha$ & $\alpha^{2}$ & $\alpha^{3}$ & $\alpha^{4}$ \\
1 & 1 & $\omega$ & $\omega^{2}$ \\
1 & $\omega$ & $\omega^{2}$ & 1 \\
1 & $\omega$ & 1 & $\omega^{2}$, \\
\hline
\end{tabular}

$$
\omega^{3}=1, \alpha^{5}=1
$$

Proceeding as in the case of the group (7), we find that

$$
(V)_{5} \equiv(V S)_{5}(\bmod .5) \text {, }
$$

$V$ being any substitution of $G$.

15. The simple groups whose orders are $<2001$ have all been determined**), as have all the primitive substitution-groups in less than 17 letters***). Our aim shall therefore be to prove, first of all, the

Theorem. With the possible exceptions of groups of orders $2^{6} \cdot 3^{4} \cdot 5$ and $2^{3} \cdot 3^{2} \cdot 5 \cdot 7$, every simple, primitive collineation-group in four variables, whose order is greater than 2000, can be represented as a substitution-group in less than 17 letters.

\section{A. The group $G=H$.}

16. On account of the property of the substitutions of $H$

$$
(T)_{p} \equiv 4(\bmod . p)
$$

*) "L-GII", § 9.

*) Hölder, Math. Ann., 40 (1892), p. 55. - Cole, American Journal of Math., 14 (1892), p. 378, and 15 (1893), p. 303. - Burnside, Proc. London Math. Soc., 26 (1895) p. 333. - Ling and Miller, American Journal of Math., 22 (1900), p. 13.

*) Jordan, Comptes Rendus, 75 (1872), p. 1754. - Miller, Quarterly Journal of Mathematics, 29 (1897), p. 225; Proc. London Math. Soc., $28(1897)$, p. 533; American Journal of Math., 20 (1898), p. 229. 
and the consequences found in "L-GII", $\S 8$, it follows that $p=2$ and that every substitution of $G$ whose order is prime to 2 is of type $(\alpha, \alpha, \beta, \beta)$. By the methods of $\$ \S 4-5$ of "L-GI" it follows that the order $g$ of $G$ contains no prime factor $>7$. By Theorems 10-13, "L-G II", we find that $g$ is not divisible by $5^{2}, 5 \cdot 7$ or $7^{2}$. It is not divisible by 9 , as no $G_{9}$ has all its substitutions of type $(\alpha, \alpha, \beta, \beta)$ (art. 11). Hence, $g$ is one of the numbers

$$
2^{a} \cdot 3,2^{a} \cdot 5,2^{a} \cdot 7,2^{a} \cdot 15,2^{a} \cdot 21
$$

The number of sub-groups of order $2^{a}$ in any of the first four cases is $<17$. In the last case, $G_{7}{ }^{\prime}=2^{b}$. Hence, a given $G_{7}$ must be transformed into itself by a certain $S_{3}$. We can have no $S_{21}$ (Theorem 11). The possibility left is that a given $S_{7}$ is changed into its second power by a $S_{3}$. The given $S_{7}$ must then be of type $\left(1, \varepsilon,-\varepsilon^{2}, \varepsilon^{4}\right), \varepsilon^{7}=1$, contrary to what is stated above concerning such a substitution. - Every group $G$ containing $H$ can therefore be represented as a substitution-group in less than 17 letters.

\section{B. The group $G$ does not contain $H$.}

17. Before proceeding to determine the orders of the groups required, we will note certain facts concerning the primitive linear homogeneous groups in 2 and 3 variables that are useful, namely*)

all such groups in 2 variables contain the similarity-substitution $(-1,-1)$

the ternary $G_{60}, G_{168}$ and $G_{360}$ contain the abelian $G_{4}$

$$
\begin{array}{r|r|r}
\hline 1 & -1 & -1 \\
-1 & -1 & 1 \\
-1 & 1 & -1 \\
\hline
\end{array}
$$

the ternary $G_{360}$ and all the remaining primitive groups in three variables must contain the similarity-substitution $(\omega, \omega, \omega)$ when written in linear homogeneous form.

Bearing in mind that the binary $G_{60}$, the ternary $G_{60}, G_{168}$ and $G_{360}$ are simple, and can therefore be generated by their substitutions of a given order $p$, we can now limit the number of cases in which two given abelian sub-groups of $G$ may have a substitution in common, using to ad-

*) We shall assume that the primitive groups in 3 variables have all been determined. See the papers by Jordan, Journal für Math., 84 (1878), p. 89; Valentiner, Kjóbenhavnske Skr. (6) Vol. 5 (1889), p. 64; Wiman, Math. Annalen 47 (1896), p. 532; and the author, "L-GI", §§ 13-17. 
vantage the principle explained in art. 14. Thus, if two abelian subgroups, $K_{1}$ and $K_{2}$, whose orders do not contain the factors 2 or 3 , have a substitution $S$ in common, then either

the group generated by $K_{1}$ and $K_{2}$ must be abelian, or

the substitution $S$ must be of type $(\alpha, \alpha, \beta, \beta)$. In this case the group generated may be intransitive:

\begin{tabular}{ll|ll}
$x$ & $y$ & $z$ & $u$ \\
\hline$\alpha$ & $\alpha$ & $\beta$ & $\beta$ \\
$\cdot$ & $\cdot$ & $\cdot$ & $\cdot$ \\
\hline
\end{tabular}

primitive in $(x, y)$ and $(z, u)$. The two latter groups are then simply isomorphic and of order ' 60 .

Suppose, for example, it possible that $K_{1}$ and $K_{2}$, both of order 25, have in common a substitution of type $\left(1, \alpha, \alpha, \alpha^{3}\right), \alpha^{5}=1$. Then, unless the group generated by $K_{1}$ and $K_{2}$ :

\begin{tabular}{l|l|ll}
$x$ & $y$ & $z$ & $u$ \\
\hline 1 & $\alpha^{3}$ & $\alpha$ & $\alpha$ \\
$\cdot$ & $\cdot$ & $\cdot$ & $\cdot$ \\
\hline
\end{tabular}

is abelian, the binary $G_{60}$ will be generated in $(z, u)$. Then we get the sub-group

$$
\begin{array}{c|c|cc}
\hline 1 & 1 & -1 & -1 \\
1 & 1 & \omega & \omega^{2} \\
\hline
\end{array}
$$$$
\omega^{3}=1
$$

from which it follows that $G$ contains $H$.

18. Besides the ordinary theorems from the theory of substitutiongroups and abstract groups, such as Sylow's Theorem (employed in art. 16), we will make use of the following Theorems of Frobenius:

I. A substitution-group of degree $n$ and of class $n-1$ is composite.*)

II. The number of substitutions of a group $G$ which satisfy the equation $S^{n}=1$ is either 1 or $k n * *$ )

A consequence of II may be noted here. Let $G$ be of order $g p^{a} q^{b}$, where $p$ and $q$ are different primes, and let there be no substitutions in $G$ of order $p q$. Then the number of substitutions in $G$ of order $p^{\alpha}(\alpha \leqq a)$ is a multiple of $q^{b}$.

We shall refer to these theorems by FI and F II.

19. Let us now consider a simple group $G$ not containing $H$. By Theorems VI, "L-GI"; 10, "L-G I" and corollaries, and art. 13, its order $g$ must be a factor of

$$
2^{6} \cdot 3^{4} \cdot 5^{3} \quad 7^{3} \cdot 11^{3} \cdot 13^{3}
$$

7) Berl. Sitzungsb. (1902), p. 455.

*) Berl. Sitzangsb. (1895), p. 988. 
If $g$ is divisible by $3^{3}$, then will $G$ contain no $S_{p}, p$ being a prime $>5$, and no $S_{5}$ which is not of type $\left(\alpha, \alpha^{2}, \alpha^{3}, \alpha^{4}\right), \alpha^{5}=1$.

Assume the contrary. Since every $G_{27}$ contains a $S_{3}$ of type $(1, \omega, \omega, \omega)$ (see art. 13) we have, by Theorem 13, "L-GII", a $S_{3_{p}}$ or a $S_{15}$ in the cases supposed. Consider the case $p=7$. A given $S_{21}$ can be written as the product of two substitutions, $S_{3}$ and $S_{7}$. If $S_{3}$ is not already of type $(1, \omega, \omega, \omega)$, it is permutable with a substitution $T_{3}$ of this type. The substitutions $T_{3}, S_{3}$ and $S_{7}$ generate an intransitive group, containing $S_{3}$ self-conjugately. It will be found, by the method given in art. 17 , that the $S_{7}$ considered must be permutable with the given $T_{3}$.

Such a substitution is always contained self-conjugately in the entire $G_{27}$. This group and the given $S_{7}$ must therefore generate an intransitive group. Taking the third type given under $(\gamma)$ in art. 13 for the $G_{27}$ considered, the group generated is of type

$$
\begin{array}{ll|lll}
x & y & z & u \\
\hline 1 & \omega & \omega & \omega \\
\omega & 1 & \omega & \omega \\
\omega & \omega & 1 & \omega \\
S_{7}: & \alpha_{1} & \alpha_{2} & \alpha_{3} & \alpha_{4}
\end{array}, \quad \alpha_{i}^{7}=1 .
$$

The group generated in $(y, z, u)$ could not be primitive (art. 17). If it were imprimitive, we would obtain an abelian $G_{63}$, generated by $T_{3}, S_{7}$ and a substitution of order 3 different from $T_{3}$. But such a group would prove the existence of the sub-group $H$ (art. 14). For, we would have a substitution of type $\left(1, \alpha, \alpha^{2}, \alpha^{4}\right), \alpha^{7}=1$; or of type

\begin{tabular}{l|l|l|l|}
$x$ & $y$ & $z$ & $u$ \\
\hline$\alpha^{4}$ & $\alpha$ & $\alpha$ & $\alpha$, \\
\hline
\end{tabular}

permutable with the substitation $T_{3}$. If the group generated in $(y, z, u)$ were intransitive, $G$ would still contain $H$.

In this manner all cases may be dealt with. There results that $g$ must be a factor of one of the numbers

$$
2^{6} \cdot 3^{2} \cdot 5^{3} \cdot 7^{3} \cdot 11^{3} \cdot 13^{3}, 2^{6} \cdot 3^{4} \cdot 5 \text {. }
$$

If $g$ is a factor of the latter, a possible $S_{5}$ of $G$ must be of type $\left(\alpha, \alpha^{2}, \alpha^{3}, \alpha^{4}\right), \alpha^{5}=1$.

20. We shall now replace these two numbers by the five

$$
2^{6} \cdot 3^{2} \cdot 5^{2}, 2^{6} \cdot 3^{2} \cdot 5 \cdot 7,2^{6} \cdot 3^{2} \cdot 5 \cdot 11,2^{6} \cdot 3^{2} \cdot 5 \cdot 13,2^{6} \cdot 3^{4} \cdot 5 \text {. }
$$

A group $G$ whose order is $2^{a} \cdot 3^{b} \cdot 5^{c} \cdot 7^{d} \cdot 11^{e} \cdot 13^{f}$ has an abelian sub-group of order $7^{d} \cdot 11^{e} \cdot 13^{f}$, - in fact it has one of order $5^{e} \cdot 7^{d} \cdot 11^{e} \cdot 13^{f}$ if $G$ contains a $S_{5}$ not of type $\left(\alpha, \alpha^{2}, \alpha^{3}, \alpha^{4}\right), \alpha^{5}=1$, which will always 
be the case when $c>1$ (Theorem 13, cor., with a slight extension, "L-G II"). Observing that no $S_{11}$ or $S_{13}$ can be of variety 2 , and therefore not of type $(\alpha, \alpha, \beta, \beta)$, by $\$ \$ 4-5$ of "L-GI", we find that, if the orders of two such sub-groups are each $>35$, then these sub-groups can have no substitution in common unless they generate an abelian group (art. 17). Now, let the greatest such sub-group in $G$ be of order $m$, and it follows by FII that $G_{m}^{\prime}=1+\mathrm{km}$. This number should be a factor of $g$.

Now, all such factors may readily be found. Assuming $m>35$, there is only one case, namely $1+11 \cdot 13=16 \cdot 9$. With the condition, just stated, that no $S_{11}$ or $S_{13}$ can be of variety 2 , we find that a subgroup of $G$ of order $11 \cdot 13$ must be of one of the following types:

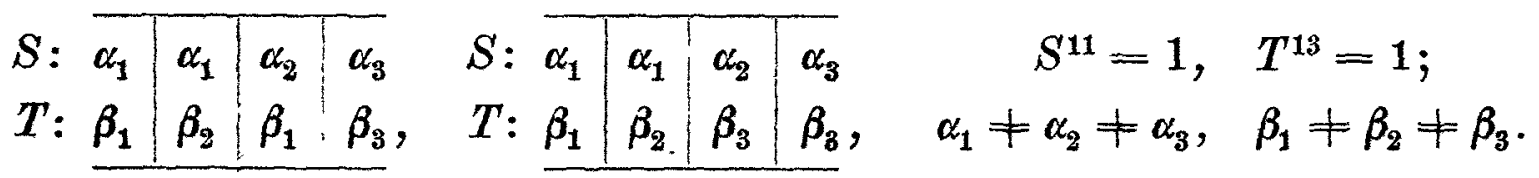

Now let us form the determinant corresponding to (3) of "L-GI", with $(V),(V S),\left(V S^{2}\right),(V T)$ and $\left(V T^{r}\right)$ for the elements of the first column, $\nabla$ being any substitution of $G$. There results

$$
\left(V T^{r}\right)_{13} \equiv(V)_{13}+r\left[(V T)_{13}-(V)_{13}\right] \quad(\bmod .13),
$$

a congruence that is either impossible or leads to the group $H$.

21. The numbers $2^{6} \cdot 3^{2} \cdot 5 \cdot 11$ and $2^{6} \cdot 3^{2} \cdot 5 \cdot 13$ may be dismissed. This is done by proving in order:

A. $G$ must contain a substitution the sum of whose multipliers is a quantity depending on $\omega$, where $\omega^{3}=1$ (i. e. the sum is of the form $a \omega+b, a \neq 0)$;

B. when such a substitution is present, $G$ must contain a $S_{33}$ or $S_{39}$, and then $G$ is not divisible by 9 ;

C. there is no simple group fulfilling these conditions.

A. Let there be no substitution $S$ such that $S=a \omega+b, a \neq 0$. Writing down all the possible weights of the substitutions of $G$, replacing their multipliers by \pm 1 or 0 , according to the rule given in the Lemma ( $\$ 3$ ) of "L-G I" (taking $p=11$ or 13), we find that they take the values $0, \pm 1, \pm 2, \pm 4$, only*). Then, according to the theory of $\$ \$ 4-5$, ibid, in order that $G$ may be primitive, it is necessary that functions exist of the form $a x^{3}+b x^{2}+c x+d, a, b, c$ not all $\equiv 0(\bmod . p)$, every one of whose remainders $(\bmod . p)$ are included in the series $0, \pm 1, \pm 2$, 14. This is found to be impossible for $p=13$. In the case $p=11$ one such function exists when $S_{11}$ is of variety 4 , namely $x^{3}-5 x$, whose

*) We must, of conrse, exclude all weights whose presence would show the existence of $H$, as for example $\left(\omega+\omega^{2}-1-1\right)$. 
remainders are $0,0,0,1,1,-1,-1,2,-2,4,-4$. These numbers, arranged in proper order, should correspond to the series of weights

$$
(V),(V S),\left(V S^{2}\right), \cdots,\left(V S^{10}\right), \quad S^{11}=1,
$$

$V$ being any given substitution of $G$ for which we do not have $V_{11} \equiv\left(V S^{i}\right)_{11}$ (mod. 11) for every value of $i$ (this could not be the case for every substitution $V$ of $G$, or this group wonld contain $H$ ).

Now, these weights are connected by the relation

$$
(V)+\alpha^{-1}(V S)+\alpha^{-2}\left(V S^{2}\right)+\cdots+\alpha^{-10}\left(V S^{10}\right)=0, \quad \alpha^{11}=1
$$

( $\$ 11$, "L-G II"). The substitution $S$ being of variety 4 , there can be no substitution of order $11 k$. Bearing this in mind, it is not very difficult to prove that (8) is an impossibility.

B. Let $T$ be a substitution, the weight of which is $a \omega+b, a \neq 0$, and $S$ a substitution of order 11 or 13 . If there are no substitutions of order 33 or 39 , we get ( $\$ 11$, "L-G II")

$$
(T)=(S T)
$$

for every substitution $S$ of order 11 or 13 , and every substitution $T$ whose weight is $a \omega+b$. Proceding as in the proof of Theorem 10; "I-G II", we find that all the substitutions of $G$ having the property enjoyed by $S$ form a group, contained self-conjugately in $G$. This group is not $=G$, as it does not contain $T^{-1}$.

Now, if $G$ contains a $S_{33}$ or $S_{39}$, then $g$ is not divisible by 9 . Otherwise we would find, by the method explained in art. 17, an abelian $G_{99}$ or $G_{117}$, from which could be shown, in any case, the presence of $H$.

C. No two sub-groups of order 33 or 39 can have a substitution in common. Hence, $G_{33}^{\prime}=1+33 k$, which does not divide $g$; and $G_{39}^{\prime}=1+39 k=40$. If $G$ contains a $S_{26}$, then it will contain the group generated by $S_{13}, S_{3}$ and $T$ :

$$
\begin{aligned}
& S_{13}: \begin{array}{llll}
x & y & z & u \\
\hline \beta_{1} & \beta_{1} & \beta_{2} & \beta_{3}
\end{array} \\
& S_{3}: \omega_{1} \quad \omega_{2}: \omega_{3} \omega_{4} \\
& T: \alpha_{1} \quad \alpha_{2} \quad \alpha_{3} \quad \alpha_{1}, \quad \beta_{i}^{13}=1, \omega_{i}^{3}=1, \alpha_{1}^{2}=\alpha_{2}^{2}=\alpha_{3}^{2}=\alpha_{4}^{2} \text {. }
\end{aligned}
$$

The group in $(x, y)$ must be imprimitive, or $G$ contains $H$. There will be at least three substitations of order 2 permutable with the given $S_{13}$, and $S_{26}^{\prime} \geqq 3 S_{13}^{\prime}$. The group $G$ contains no $S_{5 k}$. Then, by means of Sylow's and Frobenius' Theorems we prove that there is only one group possible, which is of order $2^{4} \cdot 3 \cdot 5 \cdot 13$, and only one distribution possible of its substitutions, namely 
$S_{3}^{\prime}+S_{13}^{\prime}+S_{39}^{\prime}=40 \cdot 38, \quad S_{5}^{\prime}=16 \cdot 39, \quad \sum_{i} S_{2 i}^{\prime} 25 \cdot 39, \quad S_{26}^{\prime}=S_{6}^{\prime}=S_{5 k}^{\prime}=0$.

Then, by applying the process of $\mathbf{A}$ over again to this group, we find that it cannot exist as a simple group.

22. The order of $G$ is not divisible by $5^{2}$, unless $G$ can be represented as a substitution-group in 12 or fewer letters*). - Let $g=m \cdot 5^{2}$, $m$ being a factor of $2^{6} \cdot 3^{2}$. If no two $G_{25}$ have a substitution in common, then $G_{25}^{\prime}=1+25 k=2^{6} \cdot 3^{2}$. The group can in such a case be represented as a substitution-group in $n=2^{6} \cdot 3^{2}$ letters and of class $n-1$ and is therefore not simple (FI). Hence, $G$ must contain two sub-groups of order 25 having in common a $S_{5}$. By art. 17, the two $G_{25}$ considered generate a group of order 5.60:

$$
S: \begin{array}{ll|ll}
x & y & z & u \\
\hline \alpha & \alpha & \alpha^{4} & \alpha^{4} \\
\alpha_{1} & \alpha_{2} & \alpha_{3} & \alpha_{4} \\
\alpha_{1}^{\prime} & \alpha_{2}^{\prime} & \alpha_{3}^{\prime} & \alpha_{4}^{\prime}, \quad \quad \alpha^{5}=\alpha_{i}^{5}=\left(\alpha_{i}^{\prime}\right)^{5}=1 ;
\end{array}
$$

the groups in $(x, y)$ and $(z, u)$ each being the binary $G_{60}$ and simplyisomorphic. We may suppose that

$$
\alpha_{1}=\alpha, \quad \alpha_{2}=\alpha^{4}, \quad \alpha_{3}=\alpha, \quad \alpha_{4}=\alpha^{4}
$$

or

$$
\alpha_{1}=\alpha, \quad \alpha_{2}=\alpha^{4}, \quad \alpha_{3}=\alpha^{2}, \quad \alpha_{4}=\alpha^{3} .
$$

A. Any $G_{25}$ of $G$ contains just two distinct sub-groups of type $\left(\alpha, \alpha, \alpha^{4}, \alpha^{4}\right)$. One of these will always be common to 6 groups of order 25 . If only one, the sub-groups of $G$ of order 25 fall into sets of 6 , each set containing 124 substitutions of order 5 . Hence, by F II, $\frac{124}{6} G_{25}^{\prime}=S_{5}^{\prime}=25 k-1$, or $G_{25}^{\prime}=6(1+25 l)=6$. - If each $G_{25}$ contains two sub-groups of order 5 each common to 6 groups of order 25 , then we find

$$
\left(\frac{16 \cdot 6}{2}+\frac{4+24}{7}\right) \frac{2}{6} G_{25}^{\prime}=S_{5}^{\prime}=25 k-1,
$$

or $G_{25}^{\prime}=3(12+25 l)=3 \cdot 12$. In this case $G$ contains 12 sub-groups of order 5.60. Consequently, $G$ can, in any case, be represented as a substitution-group in not more than 12 letters.

B. Here we can have only one $G_{5}$ of type $\left(\alpha, \alpha, \alpha^{4}, \alpha^{4}\right)$. We find $G_{25}^{\prime}=6(1+25 l)=6$. -

*) In the following we shall, generally speaking, dismiss all cases where $g<2000$ or where $G$ can be represented as a substitution-group in not more than 16 letters. 
To resume, the numbers that $g$ must divide are reduced to

$$
2^{6} \cdot 3^{2} \cdot 5 \cdot 7, \quad 2^{6} \cdot 3^{4} \cdot 5,
$$

with the restriction in the last case that a possible $S_{5}$ must be of type $\left(\alpha, \alpha^{2}, \alpha^{3}, \alpha^{4}\right), \alpha^{5}=1$.

23. The group $G$ does not contain substitutions of orders 35,21 or 15 . -

Suppose it contains a $G_{35}$. No two such can have a substitution of order 5 in common unless they are identical. If they have no $S_{7}$ in common, then $G_{35}^{\prime}=1+35 k$. If they have a $S_{7}$ in common, this is of type $\left(\beta, \beta, \beta^{6}, \beta^{6}\right), \beta^{7}=1$, and the two groups considered generate a $G_{7.60}$. Each $G_{7}$ will then be permutable with 6 sub-groups of $G$ of order 5 . Hence, $6 G_{7}{ }^{\prime}=G_{5}{ }^{\prime}$, and $G_{5}{ }^{\prime}=6(1+35 k)$. There being no such factor of $g$ we must have $G_{35}^{\prime}=1+35 k=36$, and $g=2^{a} \cdot 9 \cdot 5 \cdot 7$. We may assume that $a>2$, as otherwise $g<2000$. Then will $G$ contain a $S_{2}$ leaving invariant a $G_{35}$. Writing $G$ as a substitution-group in 36 letters, a $G_{35}$ will consist of a single cycle of degree 35, and powers of this cycle. It follows that we can have no $S_{2.35}$. Again, the given $S_{2}$, if it transforms a $S_{35}$ into its inverse, must be of degree 34 and is then an odd substitution, in which case $G$ is not simple. -

Let $G$ contain a $S_{21}$. If $g$ is divisible by 9 we would obtain, by the method of art. 17, an abelian $G_{63}$. No two such groups could have a substitution in common. Hence, $G_{63}^{\prime}=1+63 k=64$. Now, a $S_{5}$ could not leave invariant this $G_{63}$ unless it is permutable with it, in which case we would obtain a $S_{35}$. It follows that $g=64 \cdot 9 \cdot 7$, and $G$ is not simple (FI). Therefore $g=m \cdot 3 \cdot 7$, where $m$ is a factor of $2^{6} \cdot 5$. Now, a given $S_{3}$ can be permutable with the substitutions of only one $G_{7}$. It could not transform a $S_{7}$ into its $2^{\text {nd }}$ power, as this $S_{7}$ (and therefore every $S_{7}$ ) would in that case be of type $\left(1, \beta, \beta^{2}, \beta^{4}\right), \beta^{7}=1$, and then we could not have a $S_{21}$ as assumed. It follows that $G_{7}{ }^{\prime}=1+21 k=64$. As before, $g$ is not divisible by 5 . Hence, $g=64 \cdot 3 \cdot 7<2000$. -

Let $G$ contain a $S_{15}$. Then any given $S_{5}$ of $G$ cannot be of type $\left(\alpha, \alpha^{2}, \alpha^{3}, \alpha^{4}\right), \alpha^{5}=1$, unless $G$ contains $H$. It follows that, if $g$ is divisible by $7, G$ would contain a $S_{35}$ (Theorem 13 , "L-G II"), a case dismissed above. Therefore, $g$ is a factor of $2^{6} \cdot 3^{2} \cdot 5$. We may suppose $g=2^{6} \cdot 3^{2} \cdot 5$, as all other factors are $<2000$. Then we find that $G$ contains an abelian $G_{45}$ or a sub-group of order $3 \cdot 60$ generated by

\begin{tabular}{ll|ll}
\hline$\omega$ & $\omega$ & $\omega^{2}$ & $\omega^{2}$ \\
$\omega_{1}$ & $\omega_{2}$ & $\omega_{3}$ & $\omega_{1}$ \\
$\alpha_{1}$ & $\alpha_{2}$ & $\alpha_{3}$ & $\alpha_{4}$, \\
\hline
\end{tabular}
$\omega_{i}^{3}=1, \alpha_{i}^{5}=1$ 
If it contains an abelian $G_{45}$, we find $G_{5}{ }^{\prime}=16$. Again, the index of a $G_{180}$ is 16 . Hence, $G$ may be represented as a substitution-group in not more than 16 letters. -

24. The order of $G$ is not a factor of $2^{6} \cdot 3^{2} \cdot 5$. - We need only consider the case $g=2^{6} \cdot 3^{2} \cdot 5$. Here $G_{5}{ }^{\prime}=36$ or $=9 \cdot 64$. $G$ is composite in the latter case (FI). In the former, $G$ contains a $G_{16}$ leaving invariant a given $G_{5}$. No such group of order 16 can be constructed unless $G$ contains $H$.

25. The orders $g=64 \cdot 3 \cdot 5 \cdot 7$ and $g=32 \cdot 9 \cdot 7$ do not correspond to simple groups. - Observing that $G_{7}{ }^{\prime}$ must be even in these cases, we notice that a $G_{8}$ could not leave one $G_{7}$ invariant unless it leaves another $G_{7}$ invariant. The $G_{8}$ considered would then contain a $S_{2}$ permutable with all the substitutions of the two groups of order 7, which would, therefore, generate an intransitive group. But this would always lead to $H$. It follows that $G_{7}{ }^{\prime}=64 \cdot 15$ in the first case, and $=32.9$ in the second. Both cases are excluded by FI.

26. The order of $G$ is not $64 \cdot 5 \cdot 7$. - No number of the form $2^{a} \cdot 5$ ean be written $1+7 k$.

27. The order of $G$ is not $32 \cdot 3 \cdot 5 \cdot 7$. - We may suppose $G_{7}{ }^{\prime}=8 \cdot 15, S_{7}{ }^{\prime}=16 \cdot 45$. By FII, $G$ contains a $S_{14}$. Evidently, $S_{14}^{\prime} \geqq S_{7}{ }^{\prime}$. Moreover, $S_{5}{ }^{\prime}=64 \cdot 21$; and $G_{3}{ }^{\prime}=70,8 \cdot 35$ or $32 \cdot 35$. The last hypothesis is excluded by $F I$, and the first because no $G_{16}$ can be constructed leaving a $G_{3}$ invariant unless $G$ contains $H$. Thus, $S_{3}{ }^{\prime}=16 \cdot 35$. Then is $S_{7}^{\prime}+S_{14}^{\prime}+S_{5}^{\prime}+S_{3}^{\prime}+\sum_{i} S_{2^{i}}^{\prime}>g$, an absurdity.

28. The order of $G$ is not $2^{6} \cdot 3^{2} \cdot 7$. - In order not to get a $S_{21}$ (Theorem 13 "L-GI"), a $G_{9}$ must here be of type

\begin{tabular}{c|c|c|c}
\hline 1 & 1 & $\omega$ & $\omega^{2}$ \\
$\omega$ & $\omega^{2}$ & 1 & 1 \\
\hline
\end{tabular}

If two different $G_{9}$ have a $S_{3}$ in common, they generate an intransitive group of order $3 \cdot 12$ or $3 \cdot 24$ :

\begin{tabular}{cc:cc}
$x$ & $y$ & $z$ & $u$ \\
\hline$\omega$ & $\omega$ & $\omega^{2}$ & $\omega^{2}$ \\
$\omega$ & $\omega^{2}$ & $\omega$ & $\omega^{2}$ \\
$\omega$ & $\omega^{2}$ & $\omega$ & $\omega^{2}$, \\
\hline
\end{tabular}

the groups in $(x, y)$ and $(z, u)$ both being the binary $G_{12}$ or $G_{24}$ (primitive). We may, in any case, count the number of groups of order 9 and sub- 
stitutions of order 3 contained in $G$, as was done in art. 22 in the case of groups of order 25. We find the cases

(A) $G_{9}^{\prime}=1+9 k, \quad S_{3}^{\prime}=8 G_{9}^{\prime}$.

(B) $G_{9}{ }^{\prime}=4(1+9 k), S_{3}^{\prime}=\frac{13}{2} G_{9}{ }^{\prime}$. There is a conjugate set of $1+9 k$ groups of type $\left(\omega, \omega, \omega^{2}, \omega^{2}\right)$, each common to 4 groups of order 9 .

(C) $G_{9}{ }^{\prime}=-2+18 k, S_{3}{ }^{\prime}=5 G_{9}{ }^{\prime}$. There are $-1+9 k$ groups of type $\left(\omega, \omega, \omega^{2}, \omega^{2}\right)$, each common to 4 sub-groups of order 9 , falling in 1 or 2 conjugate sets.

A. Here $G_{9}^{\prime}=28$, and $G$ contains a $G_{16}$ leaving invariant a given $G_{9}$. This would, in all cases, prove the existence of $H$.

B. $G_{9}=16 \cdot 7$. Each of the 28 groups of type $\left(\omega, \omega, \omega^{2}, \omega^{2}\right)$ should be left invariant by a $G_{16}$ - leading to the group $H$.

C. $G_{9}{ }^{\prime}=64 \cdot 7, S_{3}{ }^{\prime}=64 \cdot 7 \cdot 5$. There are $64 \cdot 7$ substitutions of type $\left(\omega, \omega, \omega^{2}, \omega^{2}\right)$, each contained self-conjugately in a $G_{36}$ or $G_{72}$. We obtain at least $64 \cdot 7 \cdot 3$ different substitutions of order 6. Moreover, $G_{7}{ }^{\prime}=36$ or $=32 \cdot 9$. If the latter, then $S_{7}^{\prime}=32 \cdot 9 \cdot 6$, and the number of substitutions already enumerated is greater than $g$. Hence, $G_{7}{ }^{\prime}=36$. Then $G$ must contain a $G_{16}$ leaving invariant a given $G_{7}$ - resulting in the group $H$.

29. The order of $G$ is not $2^{a} \cdot 3^{2} \cdot 5 \cdot 7, a>3$. - As no number $2^{a} \cdot 9 \cdot 5$ can be written $1+7 k, G$ must contain a $S_{3}$ which transforms a $G_{7}$ into itself, without being permutable with it (art. 23). Any $S_{7}$ must therefore be of type $\left(1, \beta, \beta^{2}, \beta^{4}\right), \beta^{7}=1$. Then we can have no $S_{7 k}$ (Theorem 11, "L-GII"). Again, a $S_{5}$ must be of type $\left(\alpha, \alpha^{2}, \alpha^{3}, \alpha^{4}\right), \alpha^{5}=1$, and we can have no $S_{5 k}$. Let $a<6$. Then $G_{5}{ }^{\prime}=2.9 .7$ (F I). Hence, (FII), $a \leqq 3$, contrary to the condition given.

Let $a=6$. The enumerations given under (A), (B) and (C), art. 28, of the sub-groups of order 9 apply here.

A. $G_{9}^{\prime}=8 \cdot 35$, so that $G$ contains a $G_{8}$ leaving invariant a given $G_{9}$. As we can have no abelian $G_{18}$ (or $G$ would contain $H$ ), we find that either this $G_{8}$ will contain a substitution of type $(\alpha, \alpha, \alpha,-\alpha), \alpha^{4}=-1$, or $G$ will contain a substitution of type $\left(1,-1, \omega,-\omega^{2}\right), \omega^{3}=1$. Now, proceeding as in art. 21 (B), taking for $S$ a substitution of order 7 , we find that $G$ is not simple.

B. Here $S_{3}{ }^{\prime}=8 \cdot 35 \cdot 26$. By FII, $S_{5}{ }^{\prime}=32 \cdot 9 \cdot 7 \cdot 4$, and $S_{9}^{\prime}=64 \cdot 3 \cdot 5 \cdot{ }^{2}$., But then $S_{3}{ }^{\prime}+S_{5}{ }^{\prime}+S_{7}{ }^{\prime}>g$.

C. $G_{9}{ }^{\prime}=70$. Such a group is then left invariant by a $G_{38}$. This would prove the existence of $H$.

30. The order of $G$ is not a factor of $2^{6} \cdot 3^{3} \cdot 5$. - Let $g=m \cdot 3^{3}$, where $m$ is a factor of $2^{6} \cdot 5$. Examining all the groups of order 27 
(art. 13) we find that if two such groups (of the same type) have a substitution in common, then they will also have in common a substitution of type $(1, \omega, \omega, \omega)$, which is permutable with all the substitutions of each $G_{27}$. Now we may readily prove that $G$ would, in any case, contain $H$. It follows that $G_{27}^{\prime}=1+27 k$. But $g$ is not divisible by such a number.

It has already been proved that $g$ is not a factor of $2^{6} \cdot 3^{2} \cdot 5$ (art. 24).

31. The order of $G$ is not a factor of $2^{5} \cdot 3^{4} \cdot 5$. - We may suppose $g=2^{a} \cdot 3^{4} \cdot m, m$ being 1 or 5 . Consider the type of group of order 81 (art. 13). It possesses just one relative invariant $(x)$ of the first degree. The corresponding invariant $\left(x_{1}\right)$ of another $G_{81}$ of $G$ will be transformed by its substitutions into $9 k$ expressions, except when $x_{1}=y, z$ or $u$. It follows that if two $G_{81}$, having the same linear invariant $x$, are identical, then $G_{81}^{\prime}=1+3 l+9 k$, where $l=0$ or $=1$. Thus, under the condition stated, $G_{81}^{\prime}=4,10$ or 40 . - Let $G_{81}^{\prime}=40$. $G$ contains a $G_{2 a-3}$ leaving invariant a given $G_{81}$. This would in any case lead to $H$ if $a>4$. When $g=2^{4} \cdot 3^{4} \cdot 5, G_{5}^{\prime}=3^{4}$ or $2^{4} \cdot 3^{4}$. The first case is excluded on account of the existence of $H$; the latter by FI, as is also the case where $g=2^{3} \cdot 3^{4} \cdot 5$. Here we have $G_{81}^{\prime}=40$. No $S_{3}$ can leave a $G_{81}$ invariant unless it is contained in this $G_{81}$.

We must now examine the case when two groups of order 81 , having the same linear invariant $x$, are not identical. They will generate an intransitive group*), the systems consisting of one and three variables, say $x$, and $(y, z, u)$. If the group in $(y, z, u)$ is imprimitive, the two $G_{81}$ are identical. If the group is primitive, it must be the ternary $G_{216}$, being of order $27 n$. In this case, $G$ will contain a sub-group of order $3 \cdot 216$, of index $2^{a-3} \cdot 5$.

We may therefore assume $a=5$, and suppose $G_{3 \cdot 216}^{\prime}=20$. Each of these groups has one linear invariant. No two of these invariants are alike, or the corresponding groups would generate an intransitive group of index $\leqq 10$. Thus we have just 20 linear expressions that are transformed one into the other by the substitutions of $G$. A given $G_{81}$ leaves just one of them invariant. But this is impossible, as 20-1

32. The order of $G$ is not a factor of $2^{6} \cdot 3^{4}$. - Burnside has proved that no groups of order $p^{a} q^{b}$ can be simple, $p$ and $q$ being prime numbers (Proc. London Math. Soc., 1904, p. 388).

33. The Theorem stated in art. 15 has now been proved. Leaving out of consideration for the present possible simple groups of orders

*) See the footnote *), page 205. 
$2^{6} \cdot 3^{4} 5$ and $2^{3} \cdot 3^{2} \cdot 5 \cdot 7$ which cannot be represented as substitutiongroups in not more than 16 letters, we find, by consalting the memoirs quoted in art. 15 (footnote) that the only simple groups which need be considered are the alternating groups in $5,6,7$ and 8 letters, and the simple $G_{168}$ and $G_{504}$ (cf. articles 16,19,20 and 21). The simple $G_{504}$ may be dismissed, as it contains a cyclical $G_{9}{ }^{*}$ ). Taking the types of such groups (art. 11), we could prove, by the method of art. 21 (B), that the given $G_{504}$ should contain a $S_{21}$, which it does not (it is representable as a substitution-group in 9 letters). - There is one primitive $G_{168} * *$ ) and only one (art. 34). - There is no $G_{\frac{1}{2} 81} * *$ ). - The remaining groups are given by Maschke in Math. Ann. 51 (1899), pp. 278-292. Selecting those that are primitive we have the following types:

$22^{\circ}$. Group of order 60 generated by

$E_{1}: \varrho x^{\prime}=x, \varrho y^{\prime}=y, \varrho z^{\prime}=\omega z, \varrho u^{\prime}=\omega^{2} u, \quad \omega^{3}=1 ;$

$E_{2}: \varrho x^{\prime}=3 x, \varrho y^{\prime}=-y+2 z+2 u, \varrho z^{\prime}=2 y-z+2 u, \varrho u^{\prime}=2 y+2 z-u$; $E_{3}: \varrho x^{\prime}=-x+\sqrt{15} y, \varrho y^{\prime}=\sqrt{15} x+y, \varrho z^{\prime}=4 u, \varrho u^{\prime}=4 z$.

$23^{\circ}$. Group of order 60 generated by $E_{1}$ of $22^{\circ}$ $E_{2}: \varrho x^{\prime}=x+\sqrt{2} u, \varrho y^{\prime}=-y+\sqrt{2} z, \varrho z^{\prime}=\sqrt{2} y+z, \rho u^{\prime}=\sqrt{2} x-u$; $E_{3}: \varrho x^{\prime}=\sqrt{3} x+y, \varrho y^{\prime}=x-\sqrt{3} y, \rho z^{\prime}=2 u, \varrho u^{\prime}=2 z$.

$24^{\circ}$. Group of order 360 generated by

and

$$
E_{1}, E_{2}, E_{3} \text { of } 23^{\circ} \text {, }
$$

$$
E_{4}: \rho x^{\prime}=y, \varrho y^{\prime}=x, \rho z^{\prime}=-u, \rho u^{\prime}=-z .
$$

$25^{\circ}$. Group of order 2520 generated by

$$
\begin{aligned}
& S: \quad x^{\prime}=x, \varrho y^{\prime}=\gamma y, \varrho z^{\prime}=\gamma^{4} z, \varrho u^{\prime}=\gamma^{2} u ; \\
& W:\left\{\begin{array}{l}
\varrho x^{\prime}=p^{2} x+y+z+u, \varrho y^{\prime}=x-p y-q z-p u, \\
\varrho z^{\prime}=x-p y-p z-q u, \varrho u^{\prime}=x-q y-p z-p u ;
\end{array}\right.
\end{aligned}
$$

where

$$
p=\gamma+\gamma^{2}+\gamma^{4}, q=\gamma^{6}+\gamma^{5}+\gamma^{3}, \gamma^{7}=1 \text {. }
$$

*) Burnside, Theory of Groups, p. 374.

*) Klein; Math. Ann. 28 (1887) p. 519 gives the generating substitutions of the simple $G_{\frac{1}{2} \cdot 7 !}$ containing this $G_{168}$ as a sub-group. - See Maschke, International Math. Congress in Chicago 1893 (papers published by Macmillan and Co., 1896), p. 175.

Wiman, Math. Ann. 52 (1899), p. 243. - Maschke, Math, Ann. 51 (1899). p. 292. 
To this list we must add the following groups:

$26^{\circ}$. Group of order 168 generated by*)

$S: \rho x^{\prime}=x, \varrho y^{\prime}=\gamma y, \varrho z^{\prime}=\gamma^{4} z, \varrho u^{\prime}=\gamma^{2} u$, $\gamma^{7}=1$

$T: \varrho x^{\prime}=x, \varrho y^{\prime}=z, \varrho z^{\prime}=u, \varrho u^{\prime}=y$;

$Q:\left\{\begin{array}{l}\varrho x^{\prime}=x+y+z+u, \varrho y^{\prime}=2 x+\left(\gamma^{2}+\gamma^{5}\right) y+\left(\gamma^{3}+\gamma^{4}\right) z+\left(\gamma+\gamma^{6}\right) u \\ \varrho z^{\prime}=2 x+\left(\gamma^{3}+\gamma^{4}\right) y+\left(\gamma+\gamma^{6}\right) z+\left(\gamma^{2}+\gamma^{5}\right) u \\ \varrho u^{\prime}=2 x+\left(\gamma+\gamma^{6}\right) y+\left(\gamma^{2}+\gamma^{5}\right) z+\left(\gamma^{3}+\gamma^{4}\right) u .\end{array}\right.$

$27^{0}$. Group of order $2^{6} \cdot 3^{4} \cdot 5$ generated by**)

$A: \varrho x^{\prime}=x, \varrho y^{\prime}=z, \varrho z^{\prime}=u, \varrho u^{\prime}=y$;

$C: \varrho x^{\prime}=x, \varrho y^{\prime}=y, \varrho z^{\prime}=\omega z, \varrho u^{\prime}=\omega^{2} u$;

$D: \varrho x^{\prime}=\omega x, \varrho y^{\prime}=y, \varrho z^{\prime}=\omega z, \varrho u^{\prime}=\omega u$;

$E: \varrho x^{\prime}=\sqrt{-3} x, \varrho y^{\prime}=y+z+u, \varrho z^{\prime}=y+\omega z+\omega^{2} u, \varrho u^{\prime}=y+\omega^{2} z+\omega u$; $F: \varrho x^{\prime}=-z, \varrho y^{\prime}=y, \varrho z^{\prime}=-x, \varrho u^{\prime}=-u$.

34. All the possible types of collineation-groups in 4 variables isomorphic with the alternating groups in 5, 6 and 7 letters are included in the list $22^{\circ}-25^{\circ}$ (Cf. Maschke, 1. c.). It remains for us to prove that there' is only one type of a collineation-group isomorphic with the simple $G_{168}$, one type of order $\frac{1}{2} \cdot 7$ ! and one of order $2^{6} \cdot 3^{4} \cdot 5$, thus completing the Theorem of article 15.

Consider the simple $G_{168}$. It has a sub-group of order 21 , which must be generated by

$$
\begin{aligned}
& S: \varrho x^{\prime}=x, \varrho y^{\prime}=\gamma y, \varrho z^{\prime}=\gamma^{4} z, \varrho u^{\prime}=\gamma^{2} u, \quad \gamma^{7}=1 ; \\
& T: \varrho x^{\prime}=x, \varrho y^{\prime}=z, \varrho z^{\prime}=u, \varrho u^{\prime}=y .
\end{aligned}
$$

Choosing for new variables $z_{1}, z_{2}, z_{3}, z_{4}$, where

(9) $z_{1}=x, z_{2}=y+z+u, z_{3}=y+\omega z+\omega^{2} u, z_{4}=y+\omega^{2} z+\omega u$,

we obtain the substitutions $S$ and $T$ in the forms

(10) $S:\left\{\begin{aligned} \varrho z_{1}^{\prime} & =z_{1}, 3 \varrho z_{2}^{\prime}=p z_{2}+r z_{3}+q z_{4}, 3 \varrho z_{3}^{\prime}=q z_{2}+p z_{3}+r z_{4}, \\ 3 \varrho z_{4}^{\prime} & =r z_{2}+q z_{3}+p z_{4},\end{aligned}\right.$

*) This is the form in which it is given by Maschke, Intern. Math. Congress. in Chicago, 1893, 1. c.

*) Witting, Inaugaral-Dissertation, Göttingen (1887), p. 27, gives a set of generating substitutions for this group from which the set given here is constructed by Maschke, Math. Ann. 33 (1889), p. 320. The substitution $B$ is omitted, as $B=E^{2}$ for the collineation-group. 
where

$$
\begin{gathered}
p=\gamma+\gamma^{4}+\gamma^{2}, q=\gamma+\gamma^{4} \omega+\gamma^{2} \omega^{2}, r=\gamma+\gamma^{4} \omega^{2}+\gamma^{2} \omega \\
T: \varrho z_{1}^{\prime}=z_{1}, \varrho z_{2}^{\prime}=z_{2}, \varrho z_{3}^{\prime}=\omega^{2} z_{3}, \varrho z_{4}^{\prime}=\omega z_{4} .
\end{gathered}
$$

The group $(G)$ sought has also a substitution $Q_{1}$ of order 2 , transforming $T$ into $T^{2}$. This substitution must be, of the form

$$
Q_{1}: \varrho z_{1}^{\prime}=a z_{1}+b z_{2}, \varrho z_{2}^{\prime}=c z_{1}+d z_{2}, \varrho z_{3}^{\prime}=e z_{4}, \varrho z_{4}^{\prime}=f z_{3} \text {. }
$$

Now, $G$ contains just 8 sub-groups of order 21. With each is associated a relative linear invariant $\left(z_{1}\right.$ in $\left.(10)\right)$. No two different $G_{21}$ can have the same linear invariant, or these sub-groups would generate an intransitive $G_{168}$. Hence, the $G_{168}$ considered must transform $z_{1}$ into just eight different linear expressions. Noticing that $b \neq 0$, or $G$ would be intransitive, these 8 expressions may be written

$$
\begin{aligned}
& z_{1}, \quad Q_{1}\left(z_{1}\right) \\
& \text { (A): } \quad S Q_{1}\left(z_{1}\right), \quad T S Q_{1}\left(z_{1}\right), \quad T^{2} S Q_{1}\left(z_{1}\right) \text {; } \\
& \text { (B): } \quad S^{3} Q_{1}\left(z_{1}\right), \quad T S^{3} Q_{1}\left(z_{1}\right), \quad T^{2} S^{3} Q_{1}\left(z_{1}\right) \text {. }
\end{aligned}
$$

Writing down these expressions we find readily that $Q_{1}$ must transform any member of $(B)$ into a member of $(A)$, and vice-versa. This condition, and $Q_{1}{ }^{2}=1$, serve to determine $Q_{1}$ uniquely. We find

where

$$
a=-d=1, \quad b c=6, \quad e=q q_{1}, \quad f=r r_{1},
$$

$$
q_{1}=\gamma^{3}+\omega \gamma^{5}+\omega^{2} \gamma^{6}, \quad r_{1}=\gamma^{3}+\omega^{2} \gamma^{5}+\omega \gamma^{6} .
$$

Transforming back to the original variables $(x, y, z, u)$ we recognize the groùp $26^{\circ}$.

35. There is only one type of a collineation-group isomorphic with a simple group of order $\frac{1}{2} \cdot 7 !$. - We may assume that the group $(G)$ contains no $S_{35}$ or $S_{21}$ (cf. art. 23). We then find $G_{7}{ }^{\prime}=1+7 k=5 \cdot 3 \cdot 2^{a}$, and a given $S_{7}$ must be transformed into the $4^{\text {th }}$ power of itself by a certain $S_{3}$. We have therefore a $G_{21}$ of type (10).

The sub-group of $G$ of order 9 , to which $T$ belongs, is of type

\begin{tabular}{c|c|c|c}
$T: 1$ & 1 & $\omega$ & $\omega^{2}$ \\
$\omega$ & $\omega^{2}$ & 1 & 1 \\
\hline
\end{tabular}

This $G_{9}$ contains a $S_{3}=W_{1}$ of type $\left(\omega, \omega^{2}, 1,1\right)$, permutable with $T$ of (10), and is therefore of the form

$$
W_{1}: \rho z_{1}^{\prime}=a z_{1}+b z_{2}, \varrho z_{2}^{\prime}=c z_{1}+d z_{2}, \varrho z_{3}^{\prime}=z_{3}, \rho z_{4}^{\prime}=z_{4},
$$

where

$$
a d-b c=1, \quad a+d=\omega+\omega^{2}=-1 .
$$


Let us now construct the substitutions $S W_{1}^{*} S W_{1} T, S W_{1}^{2}$, and $S W_{1}^{2} T$. Their weights are, respectively,

$$
a+\frac{1}{3} p(d+2), \quad a+\frac{1}{3} p(d-1), \quad d+\frac{1}{3} p(a+2) \text { and } d+\frac{1}{3} p(a-1),
$$

where

Hence,

$$
p=\gamma+\gamma^{4}+\gamma^{2}
$$

$$
\begin{aligned}
& p=\left(S W_{1}\right)-\left(S W_{1} T\right)=\left(S W_{1}^{2}\right)-\left(S W_{1}^{2} T\right), \\
& p-1=\left(S W_{1}\right)+\left(S W_{1}^{2}\right) .
\end{aligned}
$$

Clearly, at least one of the substitutions $S W_{1}$ and $S W_{1} T$ must be of order 7 . We find the following possibilities:

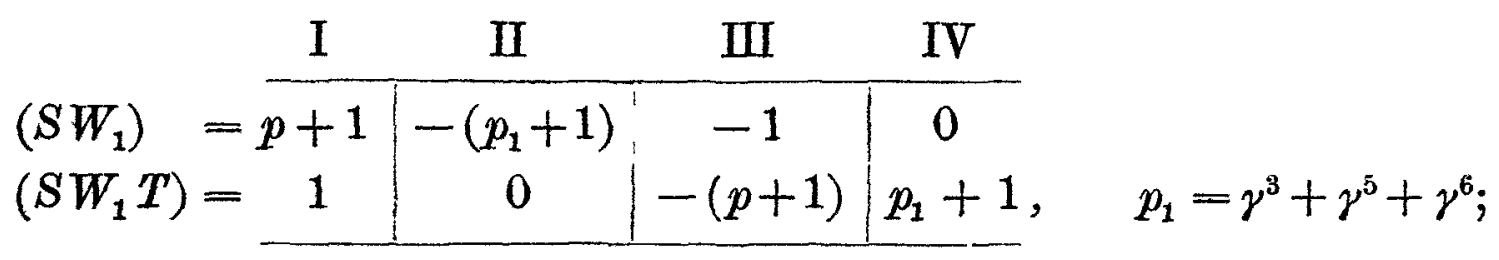

with a similar table for $\left(S W_{1}^{2}\right)$ and $\left(S W_{1}^{2} T\right)$. The condition

$$
p-1=\left(S W_{1}\right)+\left(S W_{1}^{2}\right)
$$

will exclude the cases $I$ and IV. The two remaining cases are found to be interchanged by replacing $W_{1}$ by $W_{1}{ }^{2}$. Hence, considering II only, we have $a=\frac{1}{7}(3 p-2), d=\frac{1}{7}(-3 p-5), b c=-\frac{3}{7}$. Then, transforming back to the variables $x, y, z, u$, we obtain the group given in $25^{\circ}$.

36. There is only one type of a collineation-group isomorphic with a simple group of order $2^{6} \cdot 3^{4} 5$. - Proceeding as in article 31 we find that a group $(G)$ of the order given contains either 64 sub-groups of order 81, or it must contain the Hessian $G_{216}$ as a collineation-group in 3 variables. The first hypothesis may be excluded. For, a $G_{81}$ (see $(\delta)$ of art. 13) cannot be left invariant by a $S_{5}$ unless $G$ contains $H$. Let us consider the second. The sub-group $(K)$ of $G$, of order 216 when regarded as a collineation-group in 3 variables $(y, z, u)$, must be of order $3 \cdot 216$ when considered as a collineation-group in 4 variables $(x, y, z, u)$, as it must contain the $G_{81}$ of type $(\delta)$ of article 13. Knowing its character, we may readily construct it, and find it to be generated by $(\delta)$ of article 13 and the following substitution*)

$$
\begin{gathered}
E: \varrho x^{\prime}=\left(\omega-\omega^{2}\right) x, \quad \varrho y^{\prime}=y+z+u, \quad \varrho z^{\prime}=y+\omega z+\omega^{2} u, \\
\varrho u^{\prime}=y+\omega^{2} z+\omega u .
\end{gathered}
$$

) Cf. Maschke, Math. Ann. 33, p. 324. 
Let $D$ be the substitution, contained in $(\delta)$,

$$
D: \varrho x^{\prime}=\omega x, \quad \rho y^{\prime}=y, \quad \varrho z^{\prime}=\omega z, \quad \varrho u^{\prime}=\omega u .
$$

The substitutions $E$ and $D E D^{-1}$ generate a group of order 8 . Changing the variables so that $E$ is written in canonical form, this $G_{8}$ is of type

$$
\begin{aligned}
& E: \rho x_{1}^{\prime}=i x_{1}, \quad \varrho y_{1}^{\prime}=-i y_{1}, \quad \varrho z_{1}^{\prime}=z_{1}, \quad \rho u_{1}^{\prime}=u_{1} ; \\
& D E D^{-1}: \varrho x_{1}^{\prime}=\alpha y_{1}, \quad \varrho y_{1}^{\prime}=\beta x_{1}, \quad \varrho z_{1}^{\prime}=z_{1}, \quad \varrho u_{1}^{\prime}=u_{1} .
\end{aligned}
$$

The order of $G$ being divisible by 32 , the $G_{8}$ considered is contained self-conjugately in a $G_{16}$, and this again in a $G_{32}$. By considering the different possibilities, paying attention to Theorem 10 and articles $11-12$, we find that, in all cases, the $G_{32}$ will contain a substitution $\left(M_{1}\right)$ of type

$$
M_{1}: \varrho x_{1}^{\prime}=x_{1}, \quad \varrho y_{1}^{\prime}=y_{1}, \quad \varrho z_{1}^{\prime}=i z_{1}, \quad \varrho u_{1}^{\prime}=-i u_{1} .
$$

This, written in the letters $x, y, z, u$, becomes

$M_{1}:\left\{\begin{array}{l}\varrho x^{\prime}=(q-p) x+c(z-u), \quad \varrho y^{\prime}=(q+p) y, \\ \varrho z^{\prime}=b x+p z+q u, \quad \varrho u^{\prime}=-b x+q z+p u ; \quad b c=-p^{2}-q^{2} .\end{array}\right.$

Now, with each $G_{3.216}$ is associated a single relative invariant (x) of the first degree. The number of such invariants must be of the form $1+3 k$ and is a factor of 40 , the index of $G_{3.216}$. The number is plainly 40. Taking, therefore, the letter $x$ and different expressions into which it is changed by $M_{1}$ and the substitutions of $K$, imposing the condition that there should be no more than 40) such expressions, it is found without much trouble that $q=-\omega^{2} p$ (or $=-\omega p$ ), giving the substitution

$M_{1}$ :

$$
\begin{cases}\varrho x^{\prime}=\omega x+\omega(z-u), & \varrho y^{\prime}=\left(1-\omega^{2}\right) y, \\ \varrho z^{\prime}=\omega x+z-\omega^{2} u, & \varrho u^{\prime}=-\omega x-\omega^{2} z+u .\end{cases}
$$

This is the substitution $M$ in Witting's list of generating substitutions of the known simple $G_{25920}$, from which Maschke obtains the group $27^{\circ}$ (see Math. Ann. 33, l. c.). Hence, $M_{1}$ is contained in the group $27^{\circ}$. Moreover, $K$ is the group generated by $A, C, D$ and $E$ of $27^{\circ}$, and $F=E^{3} M_{1} A E M_{1}$. The group generated by $K$ and $M_{1}$ is therefore identical with the group $27^{\circ}$. -

\section{The Primitive Groups having Invariant Primitive Sub-groups.}

37. Some of these have already been determined under $I$. and $I I$. To complete the list we must find the groups containing self-conjugately any of the types $22^{\circ}-27^{\circ}$, and the groups containing these again selfconjugately, etc. 
No substitution which is not a similarity-substitution can be permutable with every substitution of a primitive group. As a consequence, the only new groups to be added to our list, arising from the groups $22^{\circ}$, $23^{0}, 25^{\circ}$ (isomorphic with the alternating groups in 5 and 7 letters), are groups isomorphic with the symmetric groups in 5 and 7 letters*). All the types of such groups are given by Maschke in Math. Ann. 51 (1899), as is also a group isomorphic with the symmetric group in 6 letters (one type). They are as follows:

$28^{\circ}$. Group of order 120 generated by $22^{\circ}$ and the substitution

$$
F: \varrho x^{\prime}=x, \quad \varrho y^{\prime}=y, \quad \varrho z^{\prime}=u, \quad \varrho u^{\prime}=z .
$$

$29^{\circ}$. Group of order 120 generated by $23^{\circ}$ and the substitution

$$
F: \rho x^{\prime}=y, \quad \varrho y^{\prime}=-x, \quad \varrho z^{\prime}=u, \quad \varrho u^{\prime}=-z .
$$

$30^{\circ}$. Group of order 720 generated by $24^{\circ}$ and the substitution

$$
F: \varrho x^{\prime}=y, \quad \varrho y^{\prime}=-x, \varrho z^{\prime}=u, \quad \varrho u^{\prime}=-z .
$$

38. We shall now prove, in the first place, that $30^{\circ}$ is the only type of a group larger than the $G_{360}$ of $24^{\circ}$ and containing this selfconjugately; in the second place, no group larger than the $G_{720}$ of $30^{\circ}$ can contain this self-conjugately.

A simple group of order 360 contains two conjugate sets of simple groups of order 60 , each set containing 6 such groups. The group $23^{\circ}$ is a type of a $G_{60}$ of one set contained in the group of order 360 given in $24^{\circ}$, the group (95) given by Maschke in the memoir referred to in art. 37 is a type of a $G_{60}$ of the other set. These two types are not transformable one into the other (see Maschke, 1. c. p. 285). Hence, the substitutions of a group $(G)$ containing $24^{0}$ self-conjugately must leave invariant each of the sets considered. It follows that the order of $G$ must be $2^{3+a} \cdot 3^{2} \cdot 5$, and that $G$ contains a $G_{2^{2+a}}$ which leaves invariant a $G_{60}$ contained in $G$. This $G_{60}$ may be assumed to be that given in $23^{\circ}$. From what has been stated in art. 37 it follows that $a=1$ at most, that the given $G_{2^{2+\alpha}}$ and the group $23^{\circ}$ generate the group $29^{\circ}$, which is contained in the group $30^{\circ}$.

The symmetric group in 6 letters contains only one sub-group of order 360. A group containing the group $30^{\circ}$ self-conjugately must therefore contain the group $24^{\circ}$ self-conjugately, i. e. it must coincide with the group $30^{\circ}$.

39. Finally, we shall prove that a group containing either $26^{\circ}$ or $27^{\circ}$ self-eonjugately must coincide with that group.

*) Burnside, Theory of Groups, p. 246. 
Consider a group $G$ within which $K=G_{168}$ of $26^{\circ}$ is invariant. As $K$ contains 8 sub-groups of order $21, G$, if larger than $K$, must contain a substitution $(R)$, not present in $K$, which transforms a $G_{21}$ of $K$ into itself. By examining all possible cases, we find that $R$ may be assumed to be of the form

$$
\varrho x^{\prime}=a x, \quad \varrho y^{\prime}=b y, \quad \varrho z^{\prime}=c z, \quad \varrho u^{\prime}=d u,
$$

the given $G_{21}$ being generated by $S$ and $T$ of $26^{\circ}$. Now, with $K$ is associated a set of 8 linear expressions (and only one set), as was seen in art. 34. In order that this set may be left invariant by $R$ we find that $R=S^{k}$. Hence, $G$ coincides with $K$.

In the group $27^{\circ}$ of order 25920 , we have $G_{216 \cdot 3}^{\prime}=40$, and a single invariant set of 40 linear expressions (art. 36). A group $G$, containing $27^{\circ}$ self-conjugately must contain a substitution leaving invariant a given sub-group of order $3 \cdot 216$ and also the set of 40 linear expressions. We readily find that $G$ coineides with $G_{25920}$. -

40. Results for the collineation-groups in 4 variables. - A complete list of the primitive collineation-groups in 4 variables has now been obtained. Using the classification given in the introduction for such groups, we tabulate them as follows:

I. Groups having intransitive self-conjugate sub-groups, 7 types: $1^{0}-7^{0}$, articles $1-3$.

II. Groups having imprimitive self-conjugate sub-groups, 9 types: $13^{\circ}-21^{\circ}$, articles $7-8$.

III. Groups which are simple, 6 types: $22^{\circ}-27^{0}$, article 33 .

IV. Groups having primitive self-conjugate sub-groups, 8 types, distributed as follows:

5 types containing self-conjugately groups under I, namely $8^{0}-12^{0}$, article 4 ;

3 types containing self-conjugately groups under IV, namely $28^{\circ}-30^{\circ}$, article 37 .

No two of these 30 types are transformable one into the other, and any given finite, primitive collineation-group in four variables is transformable into one of these types. 